\title{
Tangence
}

\section{Le visage littéraire de la Bible}

\section{Paul-André Giguère}

Numéro 35, mars 1992

Des écritures à lire

URI : https://id.erudit.org/iderudit/025696ar

DOI : https://doi.org/10.7202/025696ar

Aller au sommaire du numéro

Éditeur(s)

Tangence

ISSN

0226-9554 (imprimé)

1710-0305 (numérique)

Découvrir la revue

Citer cet article

Giguère, P.-A. (1992). Le visage littéraire de la Bible. Tangence, (35), 24-34.

https://doi.org/10.7202/025696ar d'utilisation que vous pouvez consulter en ligne.

https://apropos.erudit.org/fr/usagers/politique-dutilisation/ 


\section{Le visage littéraire de la Bible \\ Paul-André Giguère}

De toute la production littéraire, la Bible demeure le livre le plus diffusé et aussi le plus traduit. Elle continue d'être pourtant une œuvre méconnue. J'aime en parler comme du best-seller le moins lu. On pourrait dire aussi celui qui fait l'objet du plus de lectures réductrices. Il s'agit en effet d'un de ces ouvrages que pratiquement personne n'ouvre sans une certaine idée préconçue qui commande toute lecture. Il y en a qui s'attendent à y trouver un ensemble de doctrines religieuses, d'autres une sagesse ésotérique, et d'autres, pourquoi pas, le témoignage du passage des extra-terrestres... Sa réputation de livre de sagesse religieuse qui a inspiré et guidé hommes et femmes pendant des siècles incite surtout à se la représenter comme contenant essentiellement des enseignements spirituels ou des directives morales d'une grande profondeur.

Les personnes qui ouvrent la Bible s'intéressent à son contenu, ce qui est, après tout, parfaitement normal. Très peu cependant s'intéressent à sa forme. C'est ce que nous allons faire ici.

\section{Un test de la diversité des formes littéraires}

Il suffit d'ouvrir ce gros livre au hasard à cinq endroits pour entrer immédiatement en contact avec la diversité des formes littéraires qui y sont représentées. Si vous avez une Bible à portée de la main, faites-en l'expérience. Il y a peu de chances que les cinq sections sur lesquelles vous tomberez appartiennent à la même forme, au même genre littéraire, à moins de s'en tenir aux deux grandes divisions classiques: prose ou poésie (versification serait plus exact).

Vous pouvez facilement faire un autre exercice. Essayez, à plusieurs s'il le faut, de retrouver de mémoire cinq éléments que vous savez pouvoir trouver dans la Bible. Ici encore, il y a toutes les chances que ces éléments appartiennent à des formes ou à des genres différents. Ainsi, pour en nommer parmi les plus connus, le récit d'Adam et Ève appartient au genre mythique, le 
récit de la traversée de la mer Rouge relève de l'épopée, les paroles a à chaque jour suffit sa peine * ou *il y a plus de bonheur à donner qu'à recevoir * sont évidemment des proverbes, l'histoire de l'enfant prodigue constitue une parabole.

S'étonner de cette diversité est souvent l'indice qu'on a oublié que la Bible n'est pas un livre, mais un recueil. Ce n'est pas sans raison qu'on a dit qu'elle était une bibliothèque, la plus riche certainement de toutes celles que le Proche-Orient ancien nous a laissées. On y trouve plusieurs dizaines de livres généralement indépendants les uns des autres, quoique certains aient influencé la rédaction d'écrits postérieurs et que d'autres aient fait l'objet de nombreuses relectures, comme le montrent ailleurs les articles de Jean-Jacques Lavoie et de Jérôme Longtin. Les livres contenus dans la Bible sont bien sûr de longueur inégale et ont été rédigés les uns en hébreu, les autres en grec, sur une période d'environ mille ans (grosso modo entre 950 av. J.-C. et 125 ap. J.-C.).

Ayant ainsi situé la littérature biblique, il ne sera pas nécessaire d'insister pour faire saisir qu'elle a été produite suivant les conventions littéraires de l'Antiquité orientale. Nous connaissons bien certains des genres littéraires présents dans la Bible parce qu'ils sont toujours en usage, par exemple: le proverbe, la lettre, le poème d'amour, les textes législatifs, les textes rituels, le discours patriotique. D'autres genres littéraires sont toujours vivants, mais leurs règles d'utilisation aujourd'hui sont profondément différentes de celles qui étaient acceptées à l'époque biblique. Mentionnons en particulier le récit historique et la généalogie.

Mais la plupart des genres littéraires de la Bible n'ont plus cours aujourd'hui, du moins pas en Occident. Certains sont néanmoins connus des spécialistes des littératures traditionnelles ou folkloriques, comme la saga, le récit étiologique ou l'enfance des héros. Quant au traité d'alliance de vassalité, au réquisitoire prophêtique, à l'oracle (de salut ou de condamnation), à l'évangile ou à l'apocalypse, ce sont là des genres littéraires disparus et peu ou pas connus de ceux qui ne s'intéressent pas à la littérature biblique ou aux autres littératures du Proche Orient ancien.

\section{Deux genres toujours connus: le proverbe et le chant d'amour}

Parmi les formes littéraires qui ont toujours cours aujourd'hui, regardons d'abord les proverbes. Il s'en trouve beaucoup dans la 
Bible, au point qu'un livre tout entier s'appelle précisément Livre des proverbes. C'est un écrit composite dont les parties les plus anciennes remontent aux fonctionnaires de la cour de Salomon ( $\mathrm{X}^{\circ}$ siècle av. J.-C.) et témoignent, au moins dans certaines sections, d'une nette influence des écrits de la sagesse égyptienne, en particulier la sagesse d'Amen-emopé. Les parties les plus récentes datent de la période babylonienne ( $\mathrm{VI}^{\mathrm{e}}-\mathrm{V}^{\mathrm{e}}$ siècle av. J.-C.).

Les proverbes de la Bible respectent habituellement les règles de la versification hébraïque. Il s'agit d'une poésie basée non sur la rime ou l'assonance, comme la versification classique française, mais sur le rythme marqué par les accents des mots. Le vers hébraïque est formé de deux stiques qui se correspondent avec un rythme soit égal (par exemple 3 accents +3 accents), soit inégal (par exemple $3+2$ ). En voici deux illustrations:

*Mauvais! Mauvais!* dit l'acheteur.

Mais en partant il se félicite.[...]

Le sot n'aime pas à réfléchir

mais à étaler son sentiment. (Proverbes 20,14 et 18,2).

Cette disposition que les spécialistes ont appelée "parallélisme * permet aux proverbes bibliques de développer une pensée antithétique très prononcée. La forme littéraire du proverbe incite a camper des types ou des comportements en les opposant. Une leçon de sagesse est ainsi contenue dans sa plus simple expression. Voyons-en encore quelques exemples:

Main nonchalante appauvrit, main diligente enrichit.

Amasser en été est d'un homme avisé

dormir à la moisson est d'un homme éhonté.

La mémoire du juste est en bénédiction;

le nom des méchants tombe en pourriture.

L'esprit sensé accepte les ordres,

le sot bavard court à sa perte.

Qui va franchement va sûrement,

qui use de détours est vite démasqué. (Pr 10,4-9)

Passons maintenant aux poèmes d'amour qui sont, en vérité, assez peu nombreux. "Qu'il me baise des baisers de sa bouche. Tes amours sont plus délicieuses que le vin *: voilà comment débute la célèbre collection que constitue le Cantique des cantiques. La répétition du substantif étant la formule sémitique pour exprimer le superlatif, le titre du livre signifie donc :le chant par excellence». Il s'agit là d'une suite de poèmes d'amour que 
certains auteurs croient pouvoir rattacher à une sorte de théâtre. En effet, on y trouve une alternance de monologues placés tantôt sur les lèvres d'un amant, tantôt sur celles d'une amante. Un chour de jeunes filles intervient aussi à quelques reprises, comme on en trouve régulièrement dans le théâtre antique.

Le Cantique des cantiques présente des descriptions admiratives du corps des deux amants, des expressions du désir amoureux, et surtout, peut-être, des passages marqués par l'expérience de l'alternance de la présence et de l'absence qui aiguise le désir:

Sur ma couche, de nuit, j'ai cherché celui que mon cœur aime.

Je l'ai cherché mais ne l'ai point trouvé.

Je me lèverai donc, et parcourrai la ville.

Dans les rues et sur les places,

je chercherai celui que mon cœur aime.

Je l'ai cherché, mais ne l'ai point trouvé.

Les gardes m'ont rencontrée,

ceux qui font la ronde dans la ville:

*Avez-vous vu celui que mon cœur aime?.

[...]

Je vous en conjure, filles de Jérusalem,

si vous trouvez mon bien-aimé,

que lui déclarerez-vous?

Que je suis malade d'amour.

(Cantique $3,1-3 ; 5,8$ )

\section{Un genre dont les règles ont changé: l'histoire}

Nous allons maintenant nous tourner vers les genres littéraires avec lesquels nous sommes aujourd'hui moins familiers. Et nous commencerons par le genre historique, justement parce que nous croyons le bien connaître.

Depuis près de deux siècles, l'objectivité est devenue la règle d'or des historiens. L'historien moderne n'a pas le droit de sélectionner ses sources, il doit tenir compte de toutes les sources disponibles. L'historien ne doit pas laisser ses opinions personnellés, qu'elles soient politiques, sociales ou religieuses, influencer son travail. Il n'a pas le droit d'utiliser les résultats de sa recherche à d'autres fins que la présentation objective des faits et l'interprétation de leurs causes et de leurs conséquences.

Voilà donc ce que l'on s'attend à trouver aujourd'hui dans un ouvrage d'histoire: une relation exacte et honnête, dont les conclusions ne dépassent pas ce que les faits et les documents peuvent 
supporter, qui fait bien la part des hypothèses et est honnête quant aux différents degrés de certitude dans les faits comme dans leur interprétation. Voilà pourquoi nous éprouvons souvent un certain malaise quand nous constatons que les règles étaient tout autres pour les historiens de l'Antiquité.

Pour les Anciens, la raison d'être de l'histoire était son utilité. Elle devait fournir des leçons morales, ou fonder une politique, ou susciter et nourrir la fierté patriotique. Elle savait mettre en évidence l'habileté des héros, glorifier leur courage, amplifier leurs victoires ou passer sous silence leurs défaites. Il n'en va pas autrement des écrits historiques de la Bible.

Les récits relatifs à la "conquête. de la Palestine par les Israélites et à leur installation dans le pays (essentiellement le livre de Josué et le livre des Juges) visent à établir que ce pays leur est donné par leur Dieu, Yahvé, en accomplissement de ses promesses faites à leurs ancêtres, Abraham et ses fils. Quant aux récits qui couvrent toute la période royale (1030-587 av. J.-C.) et qui remplissent les livres de Samuel et les livres des Rois, ils constituent ce que les historiens appellent l'œuvre de l'historien deutéronomiste. Ils reposent sur des sources qu'ils mentionnent à l'occasion (*les Annales des rois de Juda ", par exemple), mais impriment à leur lecture de l'histoire une coloration religieuse qui épouse les options de cet autre livre qui s'appelle le Deutéronome. C'est à la lumière des principes religieux contenus dans ce dernier, surtout le refus des cultes rendus aux autres divinités que Yahvé et l'unicité du lieu de culte à Yahvé, qu'ils jugent l'histoire et ses protagonistes, formulant pour chaque roi une appréciation de son œuvre suivant qu'il a ou non suivi ces règles.

Les profondes différences entre les présupposés et les règles des historiens anciens et des historiens modernes ont conduit certains auteurs à discréditer l'historiographie biblique comme biaisée et partiale. On n'en est plus là, maintenant que l'on connaît mieux les conventions littéraires de toute l'Antiquité. D'autre part, on ne croit plus à la supposée neutralité ou objectivité de l'historien. Dans la mesure où nous ne nous objectons plus à ce que l'on entreprenne une lecture féministe de l'histoire, par exemple, dont le projet est de réparer les oublis de l'histoire traditionnelle et de mettre en lumière le rôle et l'influence des femmes, nous avons des chances d'aborder les sections historiques de la Bible avec moins de préjugés et plus d'ouverture qu'on ne le faisait au début de ce siècle. 


\section{Une forme de récit populaire : l'étiologie}

Regardons maintenant un type particulier de récit qui n'a plus cours aujourd'hui mais qui est bien connu de ceux et celles qui étudient le folklore et la littérature orale: le récit étiologique. Mon imagination d'enfant a été beaucoup frappée par la légende du "champ de patates ", ou :les Guérets *à Rigaud. On nous racontait que cet immense champ de pierres en pleine montagne était à l'origine le champ de pommes de terre d'un cultivateur qui s'entêtait à travailler le dimanche malgré l'interdiction de l'Église. En guise de châtiment et en signe, bien sûr, d'avertissement aux autres cultivateurs, Dieu avait transformé ses pommes de terre en pierres. Combien d'autres légendes ont ainsi pris naissance pour expliquer soit des phénomènes naturels, soit des coutumes dont on ne connaissait plus l'origine ou même la signification?

On trouve un nombre assez important de semblables récits dans la Bible. Le plus connu, dans l'imaginaire des gens, est sûrement la mention de la transformation de la femme de Lot en statue de sel. Ce détail est glissé dans le récit de la destruction de Sodome et de Gomorrhe (Genèse 19,1-29), qui pourrait bien, à l'origine, être né de la curiosité soulevée par le paysage presque lunaire et volcanique du sud de la mer Morte et en particulier par un pilier rocheux ressemblant à une femme.

Un type particulier de récits étiologiques est le récit de fondation des sanctuaires. Les principaux sanctuaires palestiniens ont été le berceau de ces textes qui parlent d'une expérience du sacré vécue dans un lointain passé sur le site même. La fonction du récit est évidemment une fonction de légitimation. Le récit du songe de Jacob comme récit de fondation du sanctuaire de Béthel (Genèse 28,10-22) ou celui du passage du Jourdain à pied sec pour le sanctuaire de Gilgal (Josué 3-4; lire en particulier 4,19-24) en sont deux exemples frappants.

\section{Un genre chrétien : l'évangile}

Le genre littéraire * évangile - mérite sûrement qu'on s'y arrête à cause de l'influence considérable qu'ont eue sur la pensée religieuse et la culture occidentale les quatre écrits qui portent ce nom. Toute personne qui prend contact avec l'un ou l'autre des quatre évangiles s'imagine naturellement qu'il n'est pas autre chose qu'une biographie de Jésus de Nazareth. Deux d'entre eux ne 
commencent-ils pas par relater sa naissance et tous ne s'achèventils pas sur le long récit de son arrestation, de son procès, de son exécution publique et de sa mise au tombeau? Entre les deux, ils présentent une succession de tableaux brefs mais généralement vivants où Jésus parle, agit, voyage, recrute des disciples, les forme, soulève l'enthousiasme des foules et l'opposition des élites religieuses de son peuple.

Pourtant, les évangiles ne sont pas des biographies de Jésus. Leurs auteurs utilisent sans doute des matériaux traditionnels fiables qui nous disent vraiment quelque chose de Jésus de Nazareth et nous rapportent fidèlement certaines de ses paroles. Mais ils ne se gênent pas pour fusionner des morceaux indépendants à l'origine, situer un même événement dans des contextes différents, amplifier ou même dédoubler des récits. Aucun biographe ne peut se permettre de telles libertés. S'ils ne sont pas des biographies, que sont donc les évangiles?

Les évangiles sont des catéchèses, c'est-à-dire des enseignements destinés à soutenir les croyants dans leur foi, à les éclairer, à les instruire. Nul ne l'a mieux dit que luc dans son prologue, prologue qui obéit d'ailleurs aux conventions d'une forme littéraire connue de l'Antiquité:

Puisque beaucoup ont entrepris de composer un récit des événements qui se sont accomplis parmi nous, d'après ce que nous ont transmis ceux qui furent dès le début témoins oculaires et serviteurs de la Parole, j'ai décidé, moi aussi, après m'être informé exactement de tout depuis les origines, d'en écrire pour toi l'exposé suivi, excellent Théophile, pour que tu te rendes bien compte de la solidité des enseignements que tu as reçus (Luc 1,1-4).

À ce prologue fait écho la conclusion de l'Évangile de Jean:

Jésus a fait sous les yeux de ses disciples encore beaucoup d'autres signes, qui ne sont pas écrits dans ce livre. Ceux-là ont été mis par écrit, pour que vous croyiez que Jésus est le Christ, le Fils de Dieu, et pour qu'en croyant vous ayez la vie en son nom (Jean 20,30-31).

Catéchèses, les évangiles sont donc paroles de croyants destinées à d'autres croyants. Ils sont nés de la foi et sont destinés à la foi. Voilà d'ailleurs d'où ils tiennent leur nom, "évangile", qui n'est que la transcription dans les langues latines du grec euaggelion qui signifie • bonne nouvelle . Les Évangiles ne présentent pas Jésus de façon neutre, laissant le lecteur se faire une idée de ce qu'il fut. Ils le présentent comme *bonne nouvelle * pour l'être 
humain, en particulier pour les pauvres, les méprisés et les laissés pour compte. Les Évangiles sont nés de l'enthousiasme d'hommes et de femmes qui ont vu leur vie transformée et qui croient que la vie des autres peut l'être également.

Le genre littéraire "évangile " donne sens aux matériaux composites qu'il rassemble et qui représentent une grande variété de formes littéraires de la littérature biblique: la parabole, le discours d'exhortation, le récit de controverse, le récit de miracle, le récit de vocation, la béatitude, le proverbe, la parole de jugement, le discours apocalyptique, pour n'en nommer que quelques-unes.

\section{Un genre déroutant: l'apocalypse}

Le genre apocalyptique est pour nous l'un des plus déroutants. Apocalypse évoque pour nous catastrophe extrême, événement absolument dramatique. Pourtant, apocalypse est la transcription d'un mot grec qui signifie * révélation *. Le courant apocalyptique a été florissant du $\mathrm{II}^{\mathrm{e}}$ siècle avant J.-C. au $\mathrm{II}^{\mathrm{e}}$ siècle de notre ère, et les textes apocalyptiques que l'on retrouve dans la Bible ne représentent qu'une partie de toute la littérature qui se rattache à ce courant. Il en existe beaucoup d'autres, comme le livre d'Hénoch, l'Assomption de Moïse, le Testament des Douze patriarches... Dans la Bible, les principaux textes apocalyptiques se retrouvent aux chapitres 24 à 27 du livre d'Esaïe, 7 à 12 du livre de Daniel, 9 à 14 du livre de Zacharie, ainsi que vers la fin des évangiles. Le texte le plus connu est bien sûr l'Apocalypse de Jean, le dernier livre de la Bible chrétienne.

Nés à des périodes particulièrement troublëes de l'histoire juive et de l'histoire chrétienne primitive, ces écrits se proposent de "révéler (d'où leur nom) l'achèvement de l'histoire. Au moment où l'horizon semble bouché, où l'avenir semble compromis, les auteurs des apocalypses prétendent soulever le voile, dans une perspective religieuse, sur l'issue de l'aventure de l'humanité. Ce sont des écrits marqués par l'espérance: l'espérance de la disparition du mal et de la mort grâce à une victoire finale de Dieu sur tout ce qui s'oppose à la vie - la vie d'Israël, pour les apocalypses juives, la vie de l'Église, pour l'Apocalypse de Jean.

Paradoxalement, les apocalypses qui sont des "révélations. s'expriment de façon voilée. Leur langage est un langage codé, imagé. Presque tout y a valeur symbolique: les vêtements, les 
couleurs, les nombres, les animaux. On y déploie des scènes grandioses dans lesquelles les effets de sens s'accumulent, défiant toute cohérence rationnelle.

\section{Témoins de l'enracinement historique:}

\section{le réquisitoire prophétique et le traité d'alliance}

J'aimerais avant de conclure mentionner deux genres littéraires, inconnus aujourd'hui, qui illustrent comment la pensée religieuse juive et chrétienne est toujours enracinée dans l'histoire, jamais théorique ou intemporelle. Le premier de ces genres se retrouve dans les écrits qui remontent aux prophètes de la Bible, ces personnages qui sont la conscience du peuple. Enracinés dans la tradition religieuse d'Israël, ils interprètent l'actualité sociale, politique ou religieuse. Habités par un sens aigu de l'idéal que ce peuple s'est fixé et qui est exprimé dans la loi religieuse, ils saisissent intensément l'écart qui sépare la réalité de cet idéal.

Au lieu de formuler leur vision des choses avec l'objectivité, la neutralité ou le détachement dont essaient de faire preuve les éditorialistes et les analystes qui interprètent aujourd'hui pour nous l'actualité dans les journaux et les magazines, les prophètes s'expriment en utilisant une grande variété de formes littéraires dont une que l'on appelle le réquisitoire prophétique.

Le réquisitoire prophétique est un discours qui suit pas à pas les étapes d'une comparution juridique. Il relate un procès, un procès entre Dieu et son peuple. Pour bien apprécier l'originalité de cette forme d'expression, il convient de rappeler d'abord la façon dont se déroulaient les procès dans l'Israël ancien.

L'accusé était d'abord appelé à comparaître et l'on convoquait aussi des témoins. Mais attention: ces témoins ne sont pas ceux qui appuient l'accusation ou la défense. Ils constituent le public qui assiste à la scène, puisque déjà, dans le droit israélite, les procès devaient être publics. Le procès proprement dit commençait par l'accusation, qui était suivie immédiatement des motifs de condamnation. Il arrivait que le réquisitoire se fasse insistant pour faire ressortir la gravité de la faute, ou l'ingratitude de l'accusé face à celui qu'il avait lésé. On pouvait demander à l'accusé ce qu'il avait à dire pour sa défense. La condamnation était introduite par la formule solennelle "c'est pourquoi *. Suivait la sentence. 
À plusieurs reprises, les prophètes ont dénoncé des situations sociales ou politiques en reproduisant les formules stéréotypées du procès. En voici un exemple:

[Introduction et mention des témoins, ici la terre d'Israël]

Écoutez donc ce que dit Yahvé:

Debout! Entre en procès devant les montagnes,

et que les collines entendent ta voix!

Écoutez, montagnes, le procès de Yahvé, prêtez l'oreille, fondements de la terre,

car Yahvé est en procès avec son peuple,

il plaide contre Israël:

[Accusation implicite d'ingratitude]

Mon peuple, que t'ai-je fait? en quoi t'ai-je fatigué? Répondsmoi.

Car je t'ai fait monter du pays d'Égypte, je t'ai racheté de la maison de servitude;

j'ai envoye devant toi Moïse, Aaron et Myriam.

Mon peuple, souviens-toi donc:

quel était le projet de Balaq, roi de Moab?

Que lui répondit Balaam, fils de Béor?...

(de Shittim et de Gilgal... )

ITentative de l'accusé de se défendre et d'obtenir la faveur du juge]

Avec quoi me prosternerai-je devant Yahvé, me prosternerai-je devant le Dieu de là-haut?

Me présenterai-je avec des sacrifices, avec des veaux d'un an?

Prendra-t-il plaisir à des milliers de béliers, à des libations d'huile par torrents?

Faudra-t-il que j'offre mon aîné pour prix de mon crime, le fruit de mes entrailles pour mon propre péché?

[Sentence suspendue: une voie est offerte à l'accusé qui peut se reprendre]

On t'a fait savoir, homme, ce qui est bien, ce que Yahvé réclame de toi:

rien d'autre que d'accomplir la justice, d'aimer la bonté, et de marcher humblement avec ton Dieu.

(Michée 6,1-6; VII' siècle av. J.-C.)

Ce caractère extrêmement concret de la religion juive, qui mord dans la vie et lui emprunte même ses conventions sociales ou juridiques pour moyens d'expression, peut se retrouver encore dans la façon dont la relation avec Dieu est formulée en termes d'alliance, une alliance entre Yahvé et Israël. Et pour que ce thème religieux de la proximité et de la communauté de destin 
de Dieu et du peuple n'apparaisse pas seulement comme un enseignement désincarné, c'est en épousant les conventions des traités d'alliance tels qu'ils étaient rédigés entre deux peuples que les auteurs bibliques ont formulé leur pensée.

C'est ainsi que le cinquième livre de la Bible, le Deutéronome, est structuré exactement comme un traité d'alliance de l'époque: rappel historique (chap. 1-4), stipulation fondamentale (chap. 5), stipulations particulières (chap. 12-26,15), énumération de faveurs accompagnant le respect de l'alliance et de menaces en cas d'infidélité (chap. 28), mention de l'inscription des termes de l'alliance et d'une procédure de relecture annuelle pour la garder vivante (chap. 27). Le chapitre 24 du livre de Josué est également formulé comme les anciens traités d'alliance.

\section{En guise de conclusion}

Cet article ne pouvait faire davantage que suggérer l'étonnante diversité des genres et des formes littéraires qui sont représentés dans la Bible. Il convient d'en tirer la conclusion la plus classique de la théorie des genres littéraires: à chaque genre sa vérité. Il ne manque malheureusement pas de lecteurs modernes qui font dire toutes sortes de choses au texte de la Bible parce qu'ils ne tiennent pas compte des lois et des conventions littéraires selon lesquelles chaque texte a été rédigé. Il arrive que l'on reproche à la Bible d'affirmer des choses qu'elle n'affirme pas simplement parce qu'on manque de sensibilité littéraire. Bien sûr, on ne peut échapper à l'inévitable effet de dépaysement produit par tout contact avec une littérature étrangère, surtout quand elle est aussi ancienne et aussi différente de nos conventions et sensibilités que l'est la littérature biblique. Mais comme tout dépaysement, celui-ci est fécond et stimulant pour qui sait y consentir sans préjugés. Comme phénomène littéraire, la Bible se dresse comme un formidable monument de l'expérience humaine. Et elle témoigne que l'expérience religieuse, quand elle est authentique, assume tout ce qui est humain. 